\title{
Approximate Solution of LR Fuzzy Sylvester Matrix Equations
}

\author{
Xiaobin Guo ${ }^{1}$ and Dequan Shang ${ }^{2}$ \\ ${ }^{1}$ College of Mathematics and Statistics, Northwest Normal University, Lanzhou 730070, China \\ ${ }^{2}$ Department of Public Courses, Gansu College of Traditional Chinese Medicine, Lanzhou 730000, China
}

Correspondence should be addressed to Dequan Shang; gxbglz@163.com

Received 4 September 2012; Revised 17 October 2012; Accepted 5 November 2012

Academic Editor: Hector Pomares

Copyright (C) 2013 X. Guo and D. Shang. This is an open access article distributed under the Creative Commons Attribution License, which permits unrestricted use, distribution, and reproduction in any medium, provided the original work is properly cited.

The fuzzy Sylvester matrix equation $A \widetilde{X}+\widetilde{X} B=\widetilde{C}$ in which $A, B$ are $m \times m$ and $n \times n$ crisp matrices, respectively, and $\widetilde{C}$ is an $m \times n$ LR fuzzy numbers matrix is investigated. Based on the Kronecker product of matrices, we convert the fuzzy Sylvester matrix equation into an LR fuzzy linear system. Then we extend the fuzzy linear system into two systems of linear equations according to the arithmetic operations of LR fuzzy numbers. The fuzzy approximate solution of the original fuzzy matrix equation is obtained by solving the crisp linear systems. The existence condition of the LR fuzzy solution is also discussed. Some examples are given to illustrate the proposed method.

\section{Introduction}

System of simultaneous matrix equations plays a major role in various areas such as mathematics, physics, statistics, engineering, and social sciences. In many problems in various areas of science, which can be solved by solving a linear matrix equation, some of the system parameters are vague or imprecise, and fuzzy mathematics is better than crisp mathematics for mathematical modeling of these problems, and hence solving a linear matrix equation where some or all elements of the system are fuzzy is important. The concept of fuzzy numbers and arithmetic operations with these numbers were first introduced and investigated by Zadeh [1], Dubois and Prade [2], and Nahmias [3]. A different approach to fuzzy numbers and the structure of fuzzy number spaces was given by Puri and Ralescu [4], Goetschel and Voxman [5], and Wu and $\mathrm{Ma}[6,7]$.

Since Friedman et al. [8] proposed a general model for solving an $n \times n$ fuzzy linear systems whose coefficients matrix are crisp and the right-hand side is an arbitrary fuzzy number vector by a embedding approach, some works [920] have been done about how to deal with some advanced fuzzy linear systems such as dual fuzzy linear systems (DFLS), general fuzzy linear systems (GFLS), full fuzzy linear systems (FFLS), dual full fuzzy linear systems (DFFLS), and general dual fuzzy linear systems (GDFLS). However, for a fuzzy linear matrix equation which always has a wide use in control theory and control engineering, few works have been done in the past decades. In 2009, Allahviranloo et al. [21] studied the fuzzy linear matrix equation, (FLME) of the form $A \widetilde{X} B=\widetilde{C}$. By using the parametric form of the fuzzy number, they derived necessary and sufficient conditions for the existence of the set of fuzzy solutions and designed a numerical procedure for calculating the solutions of the fuzzy matrix equations. In 2011, Guo and Gong [22, 23] investigated a class of simple fuzzy matrix equations $A \widetilde{X}=\widetilde{B}$ by the undetermined coefficients method and studied least squares solutions of the inconsistent fuzzy matrix equation $A \widetilde{X}=\widetilde{B}$ by using generalized inverses of matrices. In 2011, Guo [24] studied the approximate solution of fuzzy Sylvester matrix equations with triangular fuzzy numbers. Lately, Guo and Shang [25] considered the fuzzy symmetric solutions of fuzzy matrix equation $A \widetilde{X}=\widetilde{B}$.

The LR fuzzy number and its arithmetic operations were first introduced by Dubois and Prade. We know that triangular fuzzy numbers are just specious cases of LR fuzzy numbers. In particular, Allahviranloo et al. [26] have showed us that the weak fuzzy solution of fuzzy linear systems $A \tilde{x}=\tilde{b}$ does not exist sometimes when $\tilde{x}, \tilde{b}$ are denoted by triangular fuzzy numbers. Recently, he also considered the LR fuzzy linear systems [27] by the linear programming 
with equality constraints. In this paper we consider the fuzzy approximate solution of LR fuzzy Sylvester matrix equation $A \widetilde{X}+\widetilde{X} B=\widetilde{C}$. In fact, the fuzzy Sylvester matrix equation $A \widetilde{X}+\widetilde{X} B=\widetilde{C}$ has numerous applications in control theory, signal processing, filtering, model reduction, decoupling techniques for ordinary and partial differential equations, and block-diagonalization of matrices and so on. But there was little research work on it. The contributions of this paper is to generalize Dubois' definition and arithmetic operation of LR fuzzy numbers and then use this result to solve fuzzy Sylvester matrix systems numerically. The structure of this paper is organized as follows.

In Section 2, we recall the LR fuzzy number, generalize the definition of LR fuzzy numbers, and present the concept of the LR fuzzy Sylvester matrix equation. The model to the fuzzy Sylvester matrix equation is proposed in detail and the fuzzy approximate solution of the original fuzzy matrix systems is derived from solving the crisp systems of linear equations in Section 3. Some examples are given in Section 4 and the conclusion is drawn in Section 5.

\section{Preliminaries}

\subsection{The LR Fuzzy Numbers}

Definition 1 (see [1]). A fuzzy number is a fuzzy set like $u$ : $R \rightarrow I=[0,1]$ which satisfies

(1) $u$ is upper semicontinuous;

(2) $u$ is fuzzy convex, that is, $u(\lambda x+(1-\lambda) y) \geq \min \{u(x)$, $u(y)\}$ for all $x, y \in R, \lambda \in[0,1]$;

(3) $u$ is normal, that is, there exists $x_{0} \in R$ such that $u\left(x_{0}\right)=1$

(4) Supp $u=\{x \in R \mid u(x)>0\}$ is the support of the $u$, and its closure $\mathrm{cl}(\operatorname{supp} u)$ is compact.

Let $E^{1}$ be the set of all fuzzy numbers on $R$.

Definition 2 (see [2]). A fuzzy number $\widetilde{M}$ is said to be an LR fuzzy number if

$$
\mu_{\widetilde{M}}(x)= \begin{cases}L\left(\frac{m-x}{\alpha}\right), & x \leq m, \alpha>0, \\ R\left(\frac{x-m}{\beta}\right), & x \geq m, \beta>0,\end{cases}
$$

where $m$ is the mean value of $\widetilde{M}$ and $\alpha$ and $\beta$ are left and right spreads, respectively. The function $L(\cdot)$ is called the left shape function of an LR fuzzy number and it satisfies:

(1) $L(x)=L(-x)$;

(2) $L(0)=1$ and $L(1)=0$;

(3) $L(x)$ is nonincreasing on $[0, \infty)$.

The definition of a right shape function $R(\cdot)$ is usually similar to that of $L(\cdot)$.

An LR fuzzy number $\widetilde{M}$ is symbolically shown as

$$
\widetilde{M}=(m, \alpha, \beta)_{\mathrm{LR}} \text {. }
$$

Clearly, $\widetilde{M}=(m, \alpha, \beta)_{\mathrm{LR}}$ is positive (negative) if and only if $m-\alpha>0(m+\beta<0)$.

Also, two LR fuzzy numbers $\widetilde{M}=(m, \alpha, \beta)_{\mathrm{LR}}$ and $\widetilde{N}=$ $(n, \gamma, \delta)_{\mathrm{LR}}$ are said to be equal, if and only if $m=n, \alpha=\gamma$, and $\beta=\delta$.

Noticing that $\alpha, \beta>0$ in Definition 2, which limits its applications, we extend the definition of LR fuzzy numbers as follows.

Definition 3 (generalized LR fuzzy numbers). (1) If $\alpha<0$ and $\beta>0$, we define $\widetilde{M}=(m, 0, \max \{-\alpha, \beta\})_{\mathrm{LR}}$ and

$$
\mu_{\widetilde{M}}(x)= \begin{cases}0, & x<m, \\ R\left(\frac{x-m}{\max \{-\alpha, \beta\}}\right), & x \geq m .\end{cases}
$$

(2) If $\alpha>0$ and $\beta<0$, we define $\widetilde{M}=(m, \max \{\alpha$, $-\beta\}, 0)_{\mathrm{LR}}$ and

$$
\mu_{\widetilde{M}}(x)= \begin{cases}L\left(\frac{x-m}{\max \{\alpha,-\beta\}}\right), & x \geq m, \\ 0, & x<m .\end{cases}
$$

(3) If $\alpha<0$ and $\beta<0$, we define $\widetilde{M}=(m,-\beta,-\alpha)_{\mathrm{LR}}$ and

$$
\mu_{\widetilde{M}}(x)= \begin{cases}L\left(\frac{m-x}{-\beta}\right), & x<m, \\ R\left(\frac{x-m}{-\alpha}\right), & x \geq m .\end{cases}
$$

Based on the extension principle, the arithmetic operations for LR fuzzy numbers were defined. For arbitrary LR fuzzy numbers $\widetilde{M}=(m, \alpha, \beta)_{\mathrm{LR}}$ and $\widetilde{N}=(n, \gamma, \delta)_{\mathrm{LR}}$, we have

(1) addition

$\widetilde{M}+\widetilde{N}=(m, \alpha, \beta)_{\mathrm{LR}}+(n, \gamma, \delta)_{\mathrm{LR}}=(m+n, \alpha+\gamma, \beta+\delta)_{\mathrm{LR}}$,

(1) scalar multiplication

$$
\begin{aligned}
\lambda \times \widetilde{M} & =\lambda \times(m, \alpha, \beta)_{\mathrm{LR}} \\
& = \begin{cases}(\lambda m, \lambda \alpha, \lambda \beta)_{\mathrm{LR}}, & \lambda>0, \\
(\lambda m,-\lambda \beta,-\lambda \alpha)_{\mathrm{RL}}, & \lambda<0 .\end{cases}
\end{aligned}
$$

2.2. Some Results on Matrix Theory. Let $A$ be an $m \times n$ real matrix and $(\cdot)^{\top}$ denote the transpose of a matrix $(\cdot)$. We recall that a generalized inverse $G$ of $A$ is an $n \times m$ matrix which satisfies one or more of Penrose equations

$$
\begin{array}{ll}
\text { (1) } A G A=A, & \text { (2) } G A G=G, \\
\text { (3) }(A G)^{\top}=A G, & \text { (4) }(G A)^{\top}=G A .
\end{array}
$$

For a subset $\{i, j, k\}$ of set $\{1,2,3,4\}$, the set of $n \times$ $m$ matrices satisfying the equations contained in $\{i, j, k\}$ is denoted by $A\{i, j, k\}$. A matrix in $A\{i, j, k\}$ is called an 
$\{i, j, k\}$-inverse of $A$ and is denoted by $A\{i, j, k\}$. In particular, the matrix $G$ is called a $\{1\}$-inverse or a $g$-inverse of $A$ if it satisfies (1). As usual, the $g$-inverse of $A$ is denoted by $A^{-}$. If $G$ satisfies (2) then it is called a $\{2\}$-inverse and If $G$ satisfies (1) and (2) then it is called a reflexive inverse or a $\{1,2\}$ inverse of $A$. The Moore-Penrose inverse of $A$ is the matrix $G$ which satisfies (1)-(4). Any matrix $A$ admits a unique MoorePenrose inverse, denoted by $A^{\dagger}$.

Lemma 4 (see [28]). For a system of linear equations

$$
A x=b .
$$

When it is consistent, its solution can be expressed by $x=G b$ in which $G \in A\{1\}$; when it has an infinite number of solutions, its minimal norm solution can be expressed by $x=G b$ in which $G \in A\{1,4\}$. When it is inconsistent, its least squares solutions can be expressed by $x=G b$ in which $G \in A\{1,3\}$. In particular, $x=A^{\dagger} b$ is the minimum norm least square solution to the above linear system.

Definition 5 (see [28]). Suppose $A=\left(a_{i j}\right) \in R^{m \times n}, B=\left(b_{i j}\right) \in$ $R^{p \times q}$; the matrix in block form

$$
A \otimes B=\left(\begin{array}{cccc}
a_{11} B & a_{12} B & \cdots & a_{1 n} B \\
a_{21} B & a_{12} B & \cdots & a_{2 n} B \\
\cdots & \cdots & \cdots & \cdots \\
a_{m 1} B & a_{m 2} B & \cdots & a_{m n} B
\end{array}\right) \in R^{m p \times n q}
$$

is said to be the Kronecker product of matrices $A$ and $B$, which denoted simply $A \otimes B=\left(a_{i j} B\right)$.

\subsection{The Fuzzy Sylvester Matrix Equation}

Definition 6. A matrix $\widetilde{A}=\left(\widetilde{a}_{i j}\right)$ is called an LR fuzzy matrix, if each element $\widetilde{a}_{i j}$ of $\widetilde{A}$ is an LR fuzzy number.

For example, we represent $m \times n$ LR fuzzy matrix $\widetilde{A}=$ $\left(\widetilde{a}_{i j}\right)$, that $\widetilde{a}_{i j}=\left(a_{i j}, \alpha_{i j}, \beta_{i j}\right)_{\mathrm{LR}}$ with new notation $\widetilde{A}=$ $(A, M, N)$, where $A=\left(a_{i j}\right), M=\left(\alpha_{i j}\right)$ and $N=\left(\beta_{i j}\right)$ are three $m \times n$ crisp matrices. Particularly, an $n$ dimensions LR fuzzy numbers vector $\tilde{x}$ can be denoted by $\left(x, x^{l}, x^{r}\right)$, where $x=\left(x_{i}\right), x^{l}=\left(x_{i}^{l}\right)$, and $x^{r}=\left(x_{i}^{r}\right)$ are three $n$ dimensions crisp vectors.

Definition 7. The matrix system

$$
\begin{gathered}
\left(\begin{array}{cccc}
a_{11} & a_{12} & \cdots & a_{1 m} \\
a_{21} & a_{12} & \cdots & a_{2 m} \\
\cdots & \cdots & \cdots & \cdots \\
a_{m n 1} & a_{m 2} & \cdots & a_{m m}
\end{array}\right)\left(\begin{array}{cccc}
\tilde{x}_{11} & \tilde{x}_{12} & \cdots & \tilde{x}_{1 n} \\
\tilde{x}_{21} & \tilde{x}_{12} & \cdots & \tilde{x}_{2 n} \\
\cdots & \cdots & \cdots & \cdots \\
\tilde{x}_{m 1} & \tilde{x}_{m 2} & \cdots & \tilde{x}_{m n}
\end{array}\right) \\
+\left(\begin{array}{cccc}
\tilde{x}_{11} & \tilde{x}_{12} & \cdots & \tilde{x}_{1 n} \\
\tilde{x}_{21} & \tilde{x}_{12} & \cdots & \tilde{x}_{2 n} \\
\cdots & \cdots & \cdots & \cdots \\
\tilde{x}_{m 1} & \tilde{x}_{m 2} & \cdots & \tilde{x}_{m n}
\end{array}\right)\left(\begin{array}{cccc}
b_{11} & b_{12} & \cdots & b_{1 n} \\
b_{21} & b_{12} & \cdots & b_{2 n} \\
\cdots & \cdots & \cdots & \cdots \\
b_{n 1} & b_{n 2} & \cdots & b_{n n}
\end{array}\right) \\
=\left(\begin{array}{cccc}
\tilde{c}_{11} & \tilde{c}_{12} & \cdots & \tilde{c}_{1 n} \\
\tilde{c}_{21} & \widetilde{c}_{12} & \cdots & \widetilde{c}_{2 n} \\
\cdots & \cdots & \cdots & \cdots \\
\tilde{c}_{m 1} & \tilde{c}_{m 2} & \cdots & \tilde{c}_{m n}
\end{array}\right),
\end{gathered}
$$

where $A$ and $B$ are real matrices and $C$ is an LR fuzzy matrix, that is, $a_{i j}, b_{i j} \in R, \widetilde{c}_{i j} \in E$, is called an LR fuzzy Sylvester matrix equations (LRFSMEs).

Using matrix notation, we have

$$
A \widetilde{X}+\widetilde{X} B=\widetilde{C} .
$$

Up to the rest of this paper, we suppose that $\widetilde{C}$ is a positive LR fuzzy numbers matrix and use the formulas given in Definition 3.

An LR fuzzy numbers matrix

$$
\widetilde{X}=\left(x_{i j}, x_{i j}^{l}, x_{i j}^{r}\right)_{\mathrm{LR}}, \quad 1 \leq i \leq m, 1 \leq j \leq n
$$

is called an LR solution of the fuzzy Sylvester matrix equation (11) if $\widetilde{X}$ satisfies (12).

\section{Method for Solving LRFSMEs}

In this section we investigate the fuzzy Sylvester matrix equations (12). Firstly, we set up a computing model for solving LRFSME. Then we define the LR fuzzy solution of LRFSME and obtain its solution representation by means of generalized inverses of matrices. Finally, we give a sufficient condition for strong fuzzy approximate solution to the fuzzy Sylvester matrix equation.

3.1. The Model. At first, we convert the fuzzy Sylvester matrix equation (12) into an LR fuzzy system of linear equations based on the Kronecker product of matrices.

Definition 8. Let $\widetilde{A}=\left(\widetilde{a}_{i j}\right)=\left(a_{i j}, a_{i j}^{l}, a_{i j}^{r}\right)_{\mathrm{LR}} \in E^{m \times n}, \widetilde{a}_{i}=$ $\left(\widetilde{a}_{1 i}, \widetilde{a}_{2 i}, \ldots, \widetilde{a}_{m i}\right)^{T}, i=1, \ldots, n$. Then the $m n$ dimensions fuzzy numbers vector

$$
\operatorname{Vec}(\widetilde{A})=\left(\begin{array}{c}
\widetilde{a}_{1} \\
\widetilde{a}_{2} \\
\vdots \\
\tilde{a}_{n}
\end{array}\right)
$$

is called the extension on column of the fuzzy matrix $\widetilde{A}$.

Theorem 9 (see [25]). Let $A=\left(a_{i j}\right)$ belong to $R^{m \times m}, \widetilde{X}=$ $\left(\tilde{x}_{i j}\right)=\left(x_{i j}, x_{i j}^{l}, x_{i j}^{r}\right)$ belong to $E^{m \times n}$, and $B=\left(b_{i j}\right)$ belong to $R^{n \times n}$. Then

$$
\operatorname{Vec}(A \widetilde{X} B)=\left(B^{T} \otimes A\right) \operatorname{Vec}(\widetilde{X})
$$

Theorem 10. Let $A=\left(a_{i j}\right)$ belong to $R^{m \times m}, \widetilde{X}=\left(\widetilde{x}_{i j}\right)=$ $\left(x_{i j}, x_{i j}^{l}, x_{i j}^{r}\right)$ belong to $E^{m \times n}$, and $B=\left(b_{i j}\right)$ belong to $R^{n \times n}$. Then

$$
\operatorname{Vec}(A \widetilde{X}+\widetilde{X} B)=\left(I_{n} \otimes A+B^{T} \otimes I_{m}\right) \operatorname{Vec}(\widetilde{X})
$$

where $I_{n}$ and $I_{m}$ denote unit matrices with order $n$ and order $m$, respectively. 
Proof. Setting $B=I_{n}$ in (15), we have

$$
\operatorname{Vec}(A \widetilde{X})=\left(I_{n} \otimes A\right) \operatorname{Vec}(\widetilde{X})
$$

Similarly, the result

$$
\operatorname{Vec}(\widetilde{X} B)=\left(B^{T} \otimes I_{m}\right) \operatorname{Vec}(\widetilde{X})
$$

is obvious when we replace $A$ with $I_{m}$ in (15).

We combine (17) and (18) and obtain the following conclusion:

$$
\begin{aligned}
\operatorname{Vec}(A \widetilde{X}+\widetilde{X} B) & =\operatorname{Vec}(A \widetilde{X})+\operatorname{Vec}(\widetilde{X} B) \\
& =\left(I_{n} \otimes A+B^{T} \otimes I_{m}\right) \operatorname{Vec}(\widetilde{X}) .
\end{aligned}
$$

Theorem 11. The matrix $\widetilde{X} \in E^{m \times n}$ is the solution of the fuzzy linear matrix equation (12) if and only if that $\tilde{x}=\operatorname{Vec}(\widetilde{X})$ is the solution of the following [LR] linear fuzzy system:

$$
G \tilde{x}=\tilde{y},
$$

where $G=\left(I_{n} \otimes A+B^{T} \otimes I_{m}\right)$ and $\widetilde{y}=\operatorname{Vec}(\widetilde{C})$.

Proof. Applying the extension operation to two sides of (12) and according to the Definition 8 and Theorem 10, we have

$$
G \tilde{x}=\tilde{y},
$$

where $G=\left(I_{n} \otimes A+B^{T} \otimes I_{m}\right)$ is an $m n \times m n$ matrix and $\widetilde{y}=\operatorname{Vec}(\widetilde{C})$ is an $m n$ LR fuzzy numbers vector. Thus the $\widetilde{X}$ is the solution of (16) is equivalent to that $\tilde{x}=\operatorname{Vec}(\widetilde{X})$ is the solution of (20).

For simplicity, we denote $p=m n$ in (20); thus

$$
G=\left(\begin{array}{cccc}
g_{11} & g_{12} & \cdots & g_{1, p} \\
g_{21} & a_{12} & \cdots & g_{2, p} \\
\cdots & \cdots & \cdots & \cdots \\
g_{p, 1} & g_{p, 2} & \cdots & g_{p, p}
\end{array}\right), \quad \tilde{y}=\left(\begin{array}{c}
\tilde{y}_{1} \\
\widetilde{y}_{2} \\
\vdots \\
\tilde{y}_{p}
\end{array}\right) \text {. }
$$

Secondly, we extend the fuzzy LR linear system (16) into two systems of linear equations according to arithmetic operations of LR fuzzy numbers.

Theorem 12. The LR fuzzy linear system (16) can be extended into the following two crisp systems of linear equations:

$$
\begin{gathered}
G x=y, \\
S\left(\begin{array}{c}
x^{l} \\
x^{r}
\end{array}\right)=\left(\begin{array}{l}
y^{l} \\
y^{r}
\end{array}\right),
\end{gathered}
$$

where $x=\left(x_{1}, x_{2}, \ldots, x_{p}\right)^{T}, x^{l}=\left(x_{1}^{l}, x_{2}^{l}, \ldots, x_{p}^{l}\right)^{T}, x^{r}=$ $\left(x_{1}^{r}, x_{2}^{r}, \ldots, x_{p}^{r}\right)^{T}, y^{l}=\left(y_{1}^{l}, y_{2}^{l}, \ldots, y_{p}^{l}\right)^{T}, y^{r}=\left(y_{1}^{r}, y_{2}^{r}, \ldots, y_{p}^{r}\right)^{T}$,
$S$ is a $2 p \times 2 p$ matrix, and $\left(s_{i j}\right), 1 \leq i, j \leq 2 p$ are determined as follows:

$$
\begin{aligned}
& g_{i j} \geq 0 \Rightarrow s_{i j}=s_{p+i, p+j}=g_{i j}, \\
& g_{i j}<0 \Rightarrow s_{i, j+p}=s_{p+i, j}=-g_{i j}, 1 \leq i, j \leq p,
\end{aligned}
$$

and any $s_{k t}$ which is not determined by the above items is zero, $1 \leq k, t \leq 2 p$.

Proof. Let $\tilde{x}=\left(\tilde{x}_{1}, \tilde{x}_{2}, \ldots, \tilde{x}_{p}\right)^{\top}, \tilde{x}_{j}=\left(x_{j}, x_{j}^{l}, x_{j}^{r}\right)_{\text {LR }}$, and let $g_{i}$ be the $i$ th row of $G, G \in \mathbb{R}^{p \times p}, g_{i}=\left(g_{i 1}, g_{i 2}, \ldots, g_{i, p}\right)$, $i=1, \ldots, p$. We can represent $G \tilde{x}$ in the form

$$
[G \tilde{x}]_{i}=g_{i} \tilde{x}, \quad i=1, \ldots, p .
$$

$$
\begin{aligned}
\text { Let } Q_{i}^{+} & =\left\{j: g_{i j} \geq 0\right\} \text { and } Q_{i}^{-}=\left\{j: g_{i j}<0\right\} \text {. We } \\
{[G \widetilde{x}]_{i} } & =\sum_{j \in Q_{i}^{+}} g_{i j} \widetilde{x}_{j}+\sum_{j \in Q_{i}^{-}} g_{i j} \widetilde{x}_{j}, \quad i=1, \ldots, p,
\end{aligned}
$$

that is,

$$
\begin{array}{r}
{[G \tilde{x}]_{i}=\left(\sum_{j \in Q_{i}^{+}} g_{i j} x_{j}+\sum_{j \in Q_{i}^{-}} g_{i j} x_{j}, \sum_{j \in Q_{i}^{+}} g_{i j} x_{j}^{l}-\sum_{j \in Q_{i}^{-}} g_{i j} x_{j}^{r},\right.} \\
\left.\sum_{j \in Q_{i}^{+}} g_{i j} x_{j}^{r}-\sum_{j \in Q_{i}^{-}} g_{i j} x_{j}^{l}\right)_{\mathrm{LR}}, \quad i=1, \ldots, p .
\end{array}
$$

Considering the given LR fuzzy vector $\tilde{y}=\left(\tilde{y}_{1}, \tilde{y}_{2}, \ldots\right.$, $\left.\tilde{y}_{p}\right)^{\top}, \tilde{y}_{i}=\left(y_{i}, y_{i}^{l}, y_{i}^{r}\right)_{\mathrm{LR}}$ for the right-hand side of (16), we can write the system (27) as

$$
\begin{gathered}
\left(g_{i} x, \sum_{j \in \mathrm{Q}_{i}^{+}} g_{i j} x_{j}^{l}-\sum_{j \in \mathrm{Q}_{i}^{-}} g_{i j} x_{j}^{r}, \sum_{j \in \mathrm{Q}_{i}^{+}} g_{i j} x_{j}^{r}-\sum_{j \in \mathrm{Q}_{i}^{-}} g_{i j} x_{j}^{l}\right)_{\mathrm{LR}} \\
=\left(y_{i}, y_{i}^{l}, y_{i}^{r}\right)_{\mathrm{LR}}, \quad i=1, \ldots, p,
\end{gathered}
$$

where $x=\left(x_{1}, \ldots, x_{p}\right)^{T}$.

Suppose $G \tilde{x}=\tilde{y}$ has a solution. Then, the corresponding mean value $x=\left(x_{1}, \ldots, x_{p}\right)^{T}$ of the solution must lie in the following crisp linear systems:

$$
G x=y,
$$

that is,

$$
\left(\begin{array}{cccc}
g_{11} & g_{12} & \cdots & g_{1, p} \\
g_{21} & a_{12} & \cdots & g_{2, p} \\
\cdots & \cdots & \cdots & \cdots \\
g_{p, 1} & g_{p, 2} & \cdots & g_{p, p}
\end{array}\right)\left(\begin{array}{c}
x_{1} \\
x_{2} \\
\vdots \\
x_{p}
\end{array}\right)=\left(\begin{array}{c}
y_{1} \\
y_{2} \\
\vdots \\
y_{p}
\end{array}\right) .
$$


Meanwhile, the left spread $x^{l}=\left(x_{1}^{l}, \ldots, x_{p}^{l}\right)^{T}$ and the right spread $x^{r}=\left(x_{1}^{r}, \ldots, x_{p}^{r}\right)^{T}$ of the solution are obtained by solving the following crisp linear systems:

$$
\left(\begin{array}{cccc}
s_{11} & s_{12} & \cdots & s_{1,2 p} \\
s_{21} & s_{12} & \cdots & s_{2,2 p} \\
\cdots & \cdots & \cdots & \cdots \\
s_{2 p, 1} & s_{2 p, 2} & \cdots & s_{2 p, 2 p}
\end{array}\right)\left(\begin{array}{c}
x_{1}^{l} \\
x_{2}^{l} \\
\vdots \\
x_{p}^{l} \\
x_{1}^{r} \\
x_{2}^{r} \\
\vdots \\
x_{p}^{r}
\end{array}\right)=\left(\begin{array}{c}
y_{1}^{l} \\
y_{2}^{l} \\
\vdots \\
y_{p}^{l} \\
y_{1}^{r} \\
y_{2}^{r} \\
\vdots \\
y_{p}^{r}
\end{array}\right),
$$

in which $\left(s_{i j}\right), 1 \leq i, j \leq 2 p$ are determined as follows:

$$
\begin{aligned}
& g_{i j} \geq 0 \Rightarrow s_{i j}=s_{p+i, p+j}=g_{i j}, \\
& g_{i j}<0 \Rightarrow s_{i, j+p}=s_{p+i, j}=-g_{i j}, 1 \leq i, j \leq p,
\end{aligned}
$$

and any $s_{k t}$ which is not determined by the above items is zero, $1 \leq k, t \leq 2 p$. Moreover, $S$ has the following structure:

$$
S=\left(\begin{array}{ll}
E & F \\
F & E
\end{array}\right) \geq 0
$$

and satisfies $G=E-F$.

3.2. Computing the Model. In order to solve the LR fuzzy Sylvester matrix equation (12), we need to consider the LR fuzzy system of linear equations (20). In order to solve (20), we need to consider the systems of linear equations (23) and (24). For instance, when matrix $G$ in (23) and matrix $S$ in (24) are both invertible, their solutions are expressed by

$$
\begin{gathered}
x=G^{-1} y, \\
\left(\begin{array}{l}
x^{l} \\
x^{r}
\end{array}\right)=S^{-1}\left(\begin{array}{l}
y^{l} \\
y^{r}
\end{array}\right),
\end{gathered}
$$

respectively.

The following Lemma shows when the matrix $S$ is nonsingular and how to calculate $S^{-1}$.

Lemma 13 (see [8]). The matrix $S$ is nonsingular if and only if the matrices $G=E-F$ and $E+F$ are both nonsingular. If $S^{-1}$ exists it must have the same structure as $S$, that is,

$$
S^{-1}=\left(\begin{array}{cc}
L & H \\
H & L
\end{array}\right),
$$

where

$$
\begin{aligned}
& L=(1 / 2)\left((E+F)^{-1}+(E-F)^{-1}\right), \\
& H=(1 / 2)\left((E+F)^{-1}-(E-F)^{-1}\right) .
\end{aligned}
$$

It seems that we have obtained the solution of the original fuzzy linear matrix system (20) as follows:

$$
\begin{aligned}
& \tilde{x}=\left(x, x^{l}, x^{r}\right)_{\mathrm{LR}}
\end{aligned}
$$

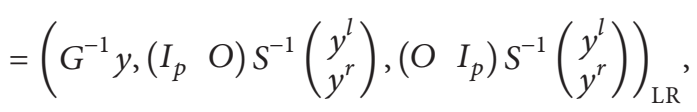

where $I_{p}$ is a $p$ order unit matrix and $O$ is a $p$ order null matrix. But the solution vector may still not be an appropriate LR fuzzy numbers vector except for $x^{l} \geq 0, x^{r} \geq 0$. So one gives the definition of LR fuzzy solutions to (11) by the fuzzy linear systems (20) as follows.

Definition 14. Let $\widetilde{x}=\left(\widetilde{x}_{1}, \widetilde{x}_{2}, \ldots, \widetilde{x}_{p}\right)^{\top}, \widetilde{x}_{j}=\left(x_{j}, x_{j}^{l}, x_{j}^{r}\right)_{\mathrm{LR}}$, $j=1, \ldots, p$. If

$$
x=\left(x_{1}, \ldots, x_{p}\right)^{\top}
$$

is an exact solution of (23), $x^{l}=\left(x_{1}^{l}, \ldots, x_{p}^{l}\right)^{\top}$ and $x^{r}=$ $\left(x_{1}^{r}, \ldots, x_{p}^{r}\right)^{\top}$ are an exact solution of (24); respectively, such that $x^{l} \geq 0, x^{r} \geq 0$, we call $\tilde{x}=\left(x, x^{l}, x^{r}\right)_{\mathrm{LR}}$ an LR fuzzy solution of (11).

When linear equation (23) or (24) is inconsistent, we can consider its approximate solution. An approximation solution which is often used is the least squares solution of (23) or (24), defined by minimizing the Frobenius norm of $(y-G x)$ or $(Y-S X)$.

For instance, we seek $X^{*} \in R^{2 p}$ to (31) such that

$$
\left\|Y-S X^{*}\right\|_{F}=\min \|Y-S X\|_{F},
$$

that is, minimizing the sum of squares of moduli of $(Y-S X)$

$$
\begin{aligned}
\|Y-S X\|_{F}^{2}=\sum_{i=1}^{P} & {\left[\left|b_{i}^{l}-\sum_{j=1}^{p}\left(s_{i j} x_{j}^{l}+s_{i, p+j} x_{j}^{r}\right)\right|^{2}\right.} \\
& \left.+\left|b_{i}^{r}-\sum_{j=1}^{p}\left(s_{p+i, j} x_{j}^{l}+s_{p+i, p+j} x_{j}^{r}\right)\right|^{2}\right] .
\end{aligned}
$$

By the generalized inverse theory [28], we know

$$
X^{*}=\left(\begin{array}{c}
x^{l} \\
x^{r}
\end{array}\right)^{*}=S^{\{1,3\}}\left(\begin{array}{c}
y^{l} \\
y^{r}
\end{array}\right),
$$

where $S^{\{1,3\}}$ is the least squares inverse of the matrix $S$.

Now we define the LR fuzzy approximate solution of the fuzzy matrix equations (11) from the fuzzy linear systems (20).

Definition 15. Let $\tilde{x}=\left(\tilde{x}_{1}, \tilde{x}_{2}, \ldots, \tilde{x}_{p}\right)^{\top}, \tilde{x}_{j}=\left(x_{j}, x_{j}^{l}, x_{j}^{r}\right)_{\mathrm{LR}}$, $j=1, \ldots, p$. If $x=\left(x_{1}, \ldots, x_{p}\right)^{T}$, or $x^{l}=\left(x_{1}^{l}, \ldots, x_{p}^{l}\right)^{T}$ and $x^{r}=\left(x_{1}^{r}, \ldots, x_{p}^{r}\right)^{T}$ are least squares solutions of $(23)$ and (24); respectively, such that $x^{l} \geq 0, x^{r} \geq 0$, then we say $\tilde{x}=\left(x, x^{l}, x^{r}\right)_{\mathrm{LR}}$ is an LR fuzzy approximate solution of (11).

Let $G$ belong to $R^{p \times p}$ and $\widetilde{y}$ be a $p$ arbitrary LR fuzzy numbers vector. Then the solutions of linear systems (23) and (24) can be expressed uniformly by

$$
\begin{gathered}
x=G^{\dagger} y, \\
\left(\begin{array}{c}
x^{l} \\
x^{r}
\end{array}\right)=S^{\dagger}\left(\begin{array}{l}
y^{l} \\
y^{r}
\end{array}\right),
\end{gathered}
$$

respectively, no matter (23) and (24) are consistent or not. 
It seems that the solution of the LR fuzzy linear system (20) can be expressed as

$$
\begin{aligned}
& \tilde{x}=\left(x, x^{l}, x^{r}\right)_{\mathrm{LR}}
\end{aligned}
$$

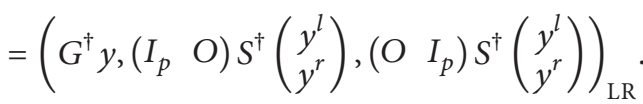

But it is not the case except for $x^{l} \geq 0$ and $x^{r} \geq 0$. In this case, we have a fuzzy set solution not fuzzy number solution. To find the fuzzy number solution, we can approximate the fuzzy set solution by a fuzzy number by one of the approximation methods finally.

Now we give a sufficient condition for LR fuzzy solution to the fuzzy Sylvester matrix equation.

3.3. A Sufficient Condition for LR Fuzzy Solution. To illustrate the expression (43) of an appropriate LR fuzzy solution vector, we now discuss the generalized inverses of matrix $S$ in a special structure.

Lemma 16 (see [18]). Let

$$
S=\left(\begin{array}{ll}
E & F \\
F & E
\end{array}\right)
$$

Then the matrix

$$
S^{\dagger}=\left(\begin{array}{cc}
(E+F)^{\dagger}+(E-F)^{\dagger} & (E+F)^{\dagger}-(E-F)^{\dagger} \\
(E+F)^{\dagger}-(E-F)^{\dagger} & (E+F)^{\dagger}+(E-F)^{\dagger}
\end{array}\right)
$$

is the Moore-Penrose inverse of the matrix $S$, where $(E+$ $F)^{\dagger},(E-F)^{\dagger}$ are Moore-Penrose inverses of matrices $E+F$ and $E-F$, respectively.

The key points to make the solution vector be an LR fuzzy solution are $x^{l} \geq 0$ and $x^{r} \geq 0$. Since $y^{l} \geq 0, y^{r} \geq 0$ and

$$
x^{l}=\left(\begin{array}{ll}
I_{p} & O
\end{array}\right) S^{\dagger}\left(\begin{array}{c}
y^{l} \\
y^{r}
\end{array}\right), \quad x^{r}=\left(\begin{array}{ll}
O & I_{p}
\end{array}\right) S^{\dagger}\left(\begin{array}{c}
y^{l} \\
y^{r}
\end{array}\right)
$$

the nonnegativity of $x^{l}$ and $x^{r}$ is equivalent to the condition $S^{\dagger} \geq 0$.

By the above analysis, one has the following conclusion.

Theorem 17. Let $G$ belong to $R^{p \times p}$ and $S^{\dagger}$ nonnegative. Then the solution of the LR fuzzy linear system (12) is expressed by

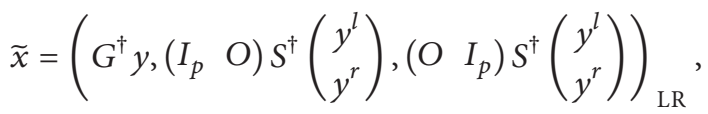

By further study, one gives a sufficient condition for obtaining nonnegative LR fuzzy solution of fuzzy Sylvester matrix equation (12) when its right-hand side $\widetilde{C}$ is a positive LR fuzzy numbers matrix.

Theorem 18. Let $G^{\dagger}$ and $S^{\dagger}$ be nonnegative matrices, and $G^{\dagger} b-\left(\begin{array}{ll}I_{p} & O\end{array}\right) S^{\dagger}\left(\begin{array}{c}y^{l} \\ y^{r}\end{array}\right) \geq 0$. Then the LR fuzzy linear system (12) has a nonnegative LR fuzzy approximate solution as follows:

$$
\left.\tilde{x}=\left(\begin{array}{ll}
G^{\dagger} b,\left(I_{p}\right. & O
\end{array}\right) S^{\dagger}\left(\begin{array}{c}
y^{l} \\
y^{r}
\end{array}\right),\left(\begin{array}{ll}
O & I_{p}
\end{array}\right) S^{\dagger}\left(\begin{array}{c}
y^{l} \\
y^{r}
\end{array}\right)\right)_{\mathrm{LR}} .
$$

Proof. Since $G^{\dagger}$ is a nonnegative matrix, we have $x=G^{\dagger} y \geq$ 0.

Now that $\left(\begin{array}{c}x^{l} \\ x^{r}\end{array}\right)=S^{\dagger}\left(\begin{array}{c}y^{l} \\ y^{r}\end{array}\right)$; therefore, with $S^{\dagger} \geq 0$ and $y^{l} \geq 0$ and $y^{r} \geq 0$, we have $\left(\begin{array}{l}x^{l} \\ x^{r}\end{array}\right) \geq 0$. Thus $\tilde{x}=\left(x, x^{l}, x^{r}\right)_{\mathrm{LR}}$ is an LR fuzzy vector which satisfies $G \tilde{x}=\tilde{y}$. Since $x-$ $x^{l}=G^{\dagger} b-\left(\begin{array}{ll}I_{p} & O\end{array}\right) S^{\dagger}\left(\begin{array}{c}y^{l} \\ y^{r}\end{array}\right) \geq 0$, the LR fuzzy linear system (12) has a nonnegative LR fuzzy approximate solution by Definition 2.

The following theorems give some results for such $S^{-1}$, $S^{\{1,3\}}$ and $S^{\dagger}$ to be nonnegative. As usual, $(\cdot)^{\top}$ denotes the transpose of a matrix $(\cdot)$.

Theorem 19 (see [29]). The inverse of a nonnegative matrix $S$ is nonnegative if and only if $S$ is a generalized permutation matrix.

Theorem 20 (see [30]). The matrix S, of rank $r$ with no zero row or zero column, admits a nonnegative $\{1,3\}$-inverse if and only if there exist some permutation matrices $P, Q$ such that

$$
P S Q=[R, *],
$$

where $R$ is a direct sum of $r$ positive, rank-one matrices.

Theorem 21 (see [29]). Let $S$ be a $2 m \times 2 n$ nonnegative matrix with rank $r$. Then the following assertions are equivalent.

(a) $S^{\dagger} \geq 0$.

(b) There exists a permutation matrix $P$, such that PS has the form

$$
P S=\left(\begin{array}{c}
B_{1} \\
B_{2} \\
\vdots \\
B_{r} \\
O
\end{array}\right)
$$

where each $B_{i}$ has rank 1 and the rows of $B_{i}$ are orthogonal to the rows of $B_{j}$, whenever $i \neq j$, the zero matrix may be absent. 
(c) $S^{\dagger}=\left(\begin{array}{cc}G C^{\top} & G D^{\top} \\ G D^{\top} & G C^{\top}\end{array}\right)$ for some positive diagonal matrix $G$. In this case,

$$
\begin{aligned}
& (C+D)^{\dagger}=G(C+D)^{\top}, \\
& (C-D)^{\dagger}=G(C-D)^{\top} .
\end{aligned}
$$

\section{Numerical Examples}

Example 22. Consider the following fuzzy matrix system:

$$
\begin{aligned}
& \left(\begin{array}{cc}
1 & 0 \\
-1 & 1
\end{array}\right)\left(\begin{array}{lll}
\tilde{x}_{11} & \tilde{x}_{12} & \tilde{x}_{13} \\
\tilde{x}_{21} & \tilde{x}_{22} & \tilde{x}_{13}
\end{array}\right) \\
& \quad+\left(\begin{array}{lll}
\tilde{x}_{11} & \tilde{x}_{12} & \tilde{x}_{13} \\
\tilde{x}_{21} & \tilde{x}_{22} & \tilde{x}_{13}
\end{array}\right)\left(\begin{array}{ccc}
1 & 0 & -1 \\
0 & -1 & 1 \\
1 & 2 & 0
\end{array}\right) \\
& =\left(\begin{array}{lll}
(3,2,1)_{\mathrm{LR}} & (4,1,1)_{\mathrm{LR}} & (2,1,1)_{\mathrm{LR}} \\
(5,2,2)_{\mathrm{LR}} & (3,1,2)_{\mathrm{LR}} & (7,2,3)_{\mathrm{LR}}
\end{array}\right) .
\end{aligned}
$$

By Theorems 9, 10, and 11, the original fuzzy matrix equation is equivalent to the following LR fuzzy linear system $G \tilde{x}=\tilde{y}$, that is,

$$
\left(\begin{array}{cccccc}
-2 & 0 & 0 & 0 & 1 & 0 \\
-1 & 1 & 0 & 0 & 0 & 1 \\
0 & 0 & 0 & 0 & 2 & 0 \\
0 & 0 & -1 & -1 & 0 & 2 \\
-1 & 0 & 1 & 0 & 1 & 0 \\
0 & -1 & 0 & 1 & -1 & 1
\end{array}\right)\left(\begin{array}{l}
\tilde{x}_{11} \\
\tilde{x}_{21} \\
\tilde{x}_{12} \\
\tilde{x}_{22} \\
\tilde{x}_{13} \\
\tilde{x}_{23}
\end{array}\right)=\left(\begin{array}{c}
(5,2,1)_{\mathrm{LR}} \\
(4,2,2)_{\mathrm{LR}} \\
(3,1,1)_{\mathrm{LR}} \\
(5,1,2)_{\mathrm{LR}} \\
(2,1,1)_{\mathrm{LR}} \\
(7,2,3)_{\mathrm{LR}}
\end{array}\right) .
$$

From Theorem 12, the model to above fuzzy linear system is made of the following two crisp systems of linear equations:

$$
\left(\begin{array}{cccccc}
-2 & 0 & 0 & 0 & 1 & 0 \\
-1 & 1 & 0 & 0 & 0 & 1 \\
0 & 0 & 0 & 0 & 2 & 0 \\
0 & 0 & -1 & -1 & 0 & 2 \\
-1 & 0 & 1 & 0 & 1 & 0 \\
0 & -1 & 0 & 1 & -1 & 1
\end{array}\right)\left(\begin{array}{l}
x_{11} \\
x_{21} \\
x_{12} \\
x_{22} \\
x_{13} \\
x_{23}
\end{array}\right)=\left(\begin{array}{l}
5 \\
4 \\
3 \\
5 \\
2 \\
7
\end{array}\right),
$$

$$
\left(\begin{array}{llllllllllll}
2 & 0 & 0 & 0 & 1 & 0 & 0 & 0 & 0 & 0 & 0 & 0 \\
0 & 1 & 0 & 0 & 0 & 1 & 1 & 0 & 0 & 0 & 0 & 0 \\
0 & 0 & 0 & 0 & 2 & 0 & 0 & 0 & 0 & 0 & 0 & 0 \\
0 & 0 & 0 & 0 & 0 & 2 & 0 & 0 & 1 & 1 & 0 & 0 \\
0 & 0 & 1 & 0 & 1 & 0 & 1 & 0 & 0 & 0 & 0 & 0 \\
0 & 0 & 0 & 1 & 0 & 1 & 0 & 1 & 0 & 0 & 1 & 0 \\
0 & 0 & 0 & 0 & 0 & 0 & 2 & 0 & 0 & 0 & 1 & 0 \\
1 & 0 & 0 & 0 & 0 & 0 & 0 & 1 & 0 & 0 & 0 & 1 \\
0 & 0 & 0 & 0 & 0 & 0 & 0 & 0 & 0 & 0 & 2 & 0 \\
0 & 0 & 1 & 1 & 0 & 0 & 0 & 0 & 0 & 0 & 0 & 2 \\
1 & 0 & 0 & 0 & 0 & 0 & 0 & 0 & 1 & 0 & 1 & 0 \\
0 & 1 & 0 & 0 & 1 & 0 & 0 & 0 & 0 & 1 & 0 & 1
\end{array}\right)\left(\begin{array}{c}
x_{11}^{l} \\
x_{21}^{l} \\
x_{12}^{l} \\
x_{22}^{l} \\
x_{13}^{l} \\
x_{23}^{l} \\
x_{11}^{r} \\
x_{21}^{r} \\
x_{12}^{r} \\
x_{22}^{r} \\
x_{13}^{r} \\
x_{23}^{r}
\end{array}\right)=\left(\begin{array}{l}
2 \\
2 \\
1 \\
1 \\
1 \\
2 \\
1 \\
2 \\
1 \\
2 \\
1 \\
3
\end{array}\right) .
$$

The coefficient matrices $G$ and $S$ are both nonsingular; we can obtain the mean value $x$, the left spread $x^{l}$, and the right spread $x^{r}$ of the solution to the above fuzzy linear system as follows:

$$
\begin{aligned}
& x=G^{-1} y \\
& =\left(\begin{array}{cccccc}
0.500 & 0 & -0.250 & 0 & 0 & 0 \\
0.500 & 0.750 & -0.500 & -0.250 & 0.250 & -0.250 \\
-0.500 & 0 & 0.750 & 0 & -1.000 & \\
0.500 & 0.500 & -0.250 & -0.500 & 0.500 & 0.500 \\
0 & 0 & 0.500 & 0 & 00 & \\
0 & 0.250 & 0.250 & 0.250 & -0.250 & 0.250
\end{array}\right) \\
& \times\left(\begin{array}{l}
5 \\
4 \\
3 \\
5 \\
2 \\
7
\end{array}\right)=\left(\begin{array}{l}
1.7500 \\
1.5000 \\
-2.250 \\
5.7500 \\
1.5000 \\
4.2500
\end{array}\right) \\
& X=\left(\begin{array}{c}
x^{l} \\
x^{r}
\end{array}\right)=S^{-1} Y=\left(\begin{array}{c}
0.7500 \\
1.3750 \\
0.2500 \\
0.5000 \\
0.5000 \\
0.3750 \\
0.2500 \\
0.6250 \\
-0.250 \\
0.5000 \\
0.5000 \\
0.6250
\end{array}\right),
\end{aligned}
$$

where 


$$
S^{-1}=\left(\begin{array}{cccccccccccc}
0.5 & 0 & -0.25 & 0 & 0 & 0 & 0 & 0 & 0 & 0 & 0 & 0 \\
-0.13 & 0.63 & -0.18 & -0.33 & 0.13 & 0.13 & -0.34 & -0.13 & -0.07 & -0.13 & 0.38 & 0.38 \\
0 & 0 & -0.5 & 0 & 1.0 & 0 & -0.5 & 0 & 0.25 & 0 & 0 & 0 \\
0.25 & -0.25 & -0.13 & -0.25 & -0.25 & 0.75 & 0.25 & -0.75 & -0.63 & 0.25 & 0.25 & 0.25 \\
0 & 0 & 0.5 & 0 & 0 & 0 & 0 & 0 & 0 & 0 & 0 & 0 \\
0.13 & 0.38 & 0.18 & 0.38 & -0.13 & -0.13 & -0.13 & 0.13 & 0.31 & 0.13 & -0.38 & -0.38 \\
0 & 0 & 0 & 0 & 0 & 0 & 0.5 & 0 & -0.25 & 0 & 0 & 0 \\
-0.38 & -0.13 & -0.06 & -0.13 & 0.38 & 0.38 & -0.13 & 0.6 & -0.18 & -0.3 & 0.13 & 0.13 \\
-0.5 & 0 & 0.25 & 0 & 0 & 0 & 0 & 0 & -0.5 & 0 & 1 & 0 \\
0.25 & -0.75 & -0.63 & 0.25 & 0.25 & 0.25 & 0.25 & -0.25 & -0.13 & -0.25 & -0.25 & 0.75 \\
0 & 0 & 0 & 0 & 0 & 0 & 0 & 0 & 0.5 & 0 & 0 & 0 \\
-0.13 & 0.13 & 0.31 & 0.13 & -0.38 & -0.38 & 0.13 & 0.38 & 0.18 & 0.38 & -0.13 & -0.13
\end{array}\right) .
$$

Since $x_{12}^{r}$ is nonpositive, the solution we obtained is not an LR fuzzy solution of the fuzzy linear system $G \tilde{x}=\tilde{y}$ and given by

$$
\tilde{x}=\left(\begin{array}{l}
\tilde{x}_{11}=(1.7500,0.7500,0.2500)_{\mathrm{LR}} \\
\tilde{x}_{21}=(1.5000,1.3750,0.6250)_{\mathrm{LR}} \\
\tilde{x}_{12}=(-2.250,0.2500,-0.250)_{\mathrm{LR}} \\
\tilde{x}_{22}=(5.7500,0.5000,0.5000)_{\mathrm{LR}} \\
\tilde{x}_{13}=(1.5000,0.5000,0.5000)_{\mathrm{LR}} \\
\tilde{x}_{23}=(4.2500,0.3750,0.6250)_{\mathrm{LR}}
\end{array}\right)
$$

$$
\widetilde{X}=\left(\begin{array}{ccc}
\tilde{x}_{11} & \tilde{x}_{12} & \tilde{x}_{13} \\
\tilde{x}_{21} & \tilde{x}_{22} & \tilde{x}_{13}
\end{array}\right)=\left(\begin{array}{ccc}
(1.750,0.750,0.250)_{\mathrm{LR}} & (-2.250,0.250,0.000)_{\mathrm{LR}} & (1.500,0.500,0.500)_{\mathrm{LR}} \\
(1.500,1.375,0.625)_{\mathrm{LR}} & (5.750,0.500,0.500)_{\mathrm{LR}} & (4.250,0.375,0.625)_{\mathrm{LR}}
\end{array}\right)
$$

According to Theorems 12 and Definitions 3 and 14, we know that the solution of the original fuzzy linear matrix equation $A \widetilde{X}+\widetilde{X} B=\widetilde{C}$ is a generalized LR fuzzy solution given by
Example 23. Consider the fuzzy Sylvester matrix system

$$
\begin{gathered}
\left(\begin{array}{cc}
-2 & 0 \\
1 & 1
\end{array}\right)\left(\begin{array}{ll}
\tilde{x}_{11} & \tilde{x}_{12} \\
\tilde{x}_{21} & \tilde{x}_{22}
\end{array}\right)+\left(\begin{array}{ll}
\tilde{x}_{11} & \tilde{x}_{12} \\
\tilde{x}_{21} & \tilde{x}_{22}
\end{array}\right)\left(\begin{array}{ll}
-1 & 1 \\
-1 & 0
\end{array}\right) \\
=\left(\begin{array}{ll}
(5,1,2)_{\mathrm{LR}} & (3,2,2)_{\mathrm{LR}} \\
(4,2,1)_{\mathrm{LR}} & (2,1,1)_{\mathrm{LR}}
\end{array}\right) .
\end{gathered}
$$

By Theorems 9, 10, and 11, the original fuzzy matrix equation is equivalent to the following LR fuzzy linear system $G \widetilde{x}=\tilde{y}$, that is,

$$
\left(\begin{array}{cccc}
-1 & 0 & 1 & 0 \\
1 & 2 & 0 & 1 \\
-1 & 0 & -2 & 0 \\
0 & -1 & 1 & 1
\end{array}\right)\left(\begin{array}{l}
\tilde{x}_{11} \\
\tilde{x}_{21} \\
\tilde{x}_{12} \\
\tilde{x}_{22}
\end{array}\right)=\left(\begin{array}{l}
(5,1,2)_{\mathrm{LR}} \\
(4,2,1)_{\mathrm{LR}} \\
(3,2,2)_{\mathrm{LR}} \\
(2,1,1)_{\mathrm{LR}}
\end{array}\right) .
$$

From Theorem 12, the model to the above fuzzy linear system is made of the following two crisp systems of linear equations:

$$
\begin{gathered}
=\left(\begin{array}{ccccc}
-1 & 0 & 1 & 0 \\
1 & 2 & 0 & 1 \\
-1 & 0 & -2 & 0 \\
0 & -1 & 1 & 1
\end{array}\right)\left(\begin{array}{l}
x_{11} \\
x_{21} \\
x_{12} \\
x_{22}
\end{array}\right)=\left(\begin{array}{l}
5 \\
4 \\
3 \\
2
\end{array}\right), \\
\left(\begin{array}{llllllll}
0 & 0 & 1 & 0 & 1 & 0 & 0 & 0 \\
1 & 2 & 0 & 1 & 0 & 0 & 0 & 0 \\
0 & 0 & 0 & 0 & 1 & 0 & 2 & 0 \\
0 & 0 & 1 & 1 & 0 & 1 & 0 & 0 \\
1 & 0 & 0 & 0 & 0 & 0 & 1 & 0 \\
0 & 0 & 0 & 0 & 1 & 2 & 0 & 1 \\
1 & 0 & 2 & 0 & 0 & 0 & 0 & 0 \\
0 & 1 & 0 & 0 & 0 & 0 & 1 & 1
\end{array}\right)\left(\begin{array}{c}
x_{11}^{l} \\
x_{21}^{l} \\
x_{12}^{l} \\
x_{22}^{l} \\
x_{11}^{r} \\
x_{21}^{r} \\
x_{12}^{r} \\
x_{22}^{r}
\end{array}\right)=\left(\begin{array}{l}
1 \\
2 \\
2 \\
1 \\
2 \\
1 \\
2 \\
1
\end{array}\right) .
\end{gathered}
$$

The coefficient matrices $G$ and $S$ are both singular; we can obtain the mean value $x$, the left spread $x^{l}$, and the right 
spread $x^{r}$ of the solution to the above fuzzy linear system by the same method

$$
x=G^{\dagger} y=\left(\begin{array}{c}
-4.3333 \\
2.3333 \\
0.6667 \\
3.6667
\end{array}\right), \quad\left(\begin{array}{c}
x^{l} \\
x^{r}
\end{array}\right)=S^{\dagger} Y=\left(\begin{array}{l}
1.333 \\
0.000 \\
0.333 \\
0.667 \\
0.667 \\
0.000 \\
0.667 \\
0.333
\end{array}\right) \text {. }
$$

Since $x_{i j}^{l}, x_{i j}^{r}, i, j=1,2$ are nonnegative, the solution we obtained is an LR fuzzy approximate solution of the fuzzy linear system $G \tilde{x}=\tilde{y}$ and given by

$$
\tilde{x}=\left(\begin{array}{c}
\tilde{x}_{11}=(-4.3333,1.3333,0.6667)_{\mathrm{LR}} \\
\tilde{x}_{21}=(2.3333,0.0000,0.0000)_{\mathrm{LR}} \\
\tilde{x}_{12}=(0.6667,0.3333,0.6667)_{\mathrm{LR}} \\
\tilde{x}_{22}=(3.6667,0.6667,0.3333)_{\mathrm{LR}}
\end{array}\right) .
$$

According to Theorems 12 and Definition 15, we know that the fuzzy approximate solution of the original fuzzy linear matrix equation $A \widetilde{X}+\widetilde{X} B=\widetilde{C}$ is

$$
\begin{aligned}
\widetilde{X} & =\left(\begin{array}{ll}
\tilde{x}_{11} & \tilde{x}_{12} \\
\tilde{x}_{21} & \tilde{x}_{22}
\end{array}\right) \\
& =\left(\begin{array}{l}
(-4.3333,1.3333,0.6667)_{\mathrm{LR}}(0.6667,0.3333,0.6667)_{\mathrm{LR}} \\
(2.3333,0.0000,0.0000)_{\mathrm{LR}}
\end{array}\right),
\end{aligned}
$$

and it admits an appropriate LR fuzzy approximate solution.

\section{Conclusion}

In this work we presented a model for solving fuzzy Sylvester matrix equations $A \widetilde{X}+\widetilde{X} B=\widetilde{C}$ where $A$ and $B$ are $m \times m$ and $n \times n$ crisp matrices, respectively, and $\widetilde{C}$ is an $m \times n$ arbitrary LR fuzzy numbers matrix. The model was proposed in this way, that is, we converted the fuzzy linear matrix equation into an LR fuzzy linear systems, then we extended the fuzzy linear systems into two systems of linear equations. The LR fuzzy solution of the fuzzy matrix equation was derived from solving the crisp systems of linear equations. In addition, the existence condition of LR fuzzy solution was studied. Numerical examples showed that our method is feasible to solve this type of fuzzy matrix equations.

\section{Acknowledgments}

The authors were very thankful for the reviewer's helpful suggestions to improve the paper. This paper was financially supported by the National Natural Science Foundation of China (71061013) and the Youth Scientific Research Ability Promotion Project of Northwest Normal University (nwnulkqn-11-20).

\section{References}

[1] L. A. Zadeh, "The concept of a linguistic variable and its application to approximate reasoning. I," vol. 8, pp. 199-249, 1975.

[2] D. Dubois and H. Prade, "Operations on fuzzy numbers," International Journal of Systems Science, vol. 9, no. 6, pp. 613626, 1978.

[3] S. Nahmias, "Fuzzy variables," Fuzzy Sets and Systems, vol. 1, no. 2, pp. 97-110, 1978.

[4] M. L. Puri and D. A. Ralescu, "Differentials of fuzzy functions," Journal of Mathematical Analysis and Applications, vol. 91, no. 2, pp. 552-558, 1983.

[5] R. Goetschel Jr. and W. Voxman, "Elementary fuzzy calculus," Fuzzy Sets and Systems, vol. 18, no. 1, pp. 31-43, 1986.

[6] C. X. Wu and M. Ma, "Embedding problem of fuzzy number space. I," Fuzzy Sets and Systems, vol. 44, no. 1, pp. 33-38, 1991.

[7] C. X. Wu and M. Ma, "Embedding problem of fuzzy number space. III," Fuzzy Sets and Systems, vol. 46, no. 2, pp. 281-286, 1992.

[8] M. Friedman, M. Ming, and A. Kandel, "Fuzzy linear systems," Fuzzy Sets and Systems, vol. 96, no. 2, pp. 201-209, 1998.

[9] M. Ma, M. Friedman, and A. Kandel, "Duality in fuzzy linear systems," Fuzzy Sets and Systems, vol. 109, no. 1, pp. 55-58, 2000.

[10] T. Allahviranloo, "Numerical methods for fuzzy system of linear equations," Applied Mathematics and Computation, vol. 155, no. 2, pp. 493-502, 2004.

[11] T. Allahviranloo, "Successive over relaxation iterative method for fuzzy system of linear equations," Applied Mathematics and Computation, vol. 162, no. 1, pp. 189-196, 2005.

[12] T. Allahviranloo, "The Adomian decomposition method for fuzzy system of linear equations," Applied Mathematics and Computation, vol. 163, no. 2, pp. 553-563, 2005.

[13] S. Abbasbandy, R. Ezzati, and A. Jafarian, " $L U$ decomposition method for solving fuzzy system of linear equations," Applied Mathematics and Computation, vol. 172, no. 1, pp. 633-643, 2006.

[14] S. Abbasbandy, A. Jafarian, and R. Ezzati, "Conjugate gradient method for fuzzy symmetric positive definite system of linear equations," Applied Mathematics and Computation, vol. 171, no. 2, pp. 1184-1191, 2005

[15] S. Abbasbandy, M. Otadi, and M. Mosleh, "Minimal solution of general dual fuzzy linear systems," Chaos, Solitons \& Fractals, vol. 37, no. 4, pp. 1113-1124, 2008.

[16] B. Asady, S. Abbasbandy, and M. Alavi, "Fuzzy general linear systems," Applied Mathematics and Computation, vol. 169, no. 1, pp. 34-40, 2005.

[17] K. Wang and B. Zheng, "Inconsistent fuzzy linear systems," Applied Mathematics and Computation, vol. 181, no. 2, pp. 973981, 2006.

[18] B. Zheng and K. Wang, "General fuzzy linear systems," Applied Mathematics and Computation, vol. 181, no. 2, pp. 1276-1286, 2006.

[19] M. Dehghan, B. Hashemi, and M. Ghatee, "Computational methods for solving fully fuzzy linear systems," Applied Mathematics and Computation, vol. 179, no. 1, pp. 328-343, 2006.

[20] M. Dehghan, B. Hashemi, and M. Ghatee, "Solution of the fully fuzzy linear systems using iterative techniques," Chaos, Solitons \& Fractals, vol. 34, no. 2, pp. 316-336, 2007.

[21] T. Allahviranloo, N. Mikaeilvand, and M. Barkhordary, "Fuzzy linear matrix equation," Fuzzy Optimization and Decision Making, vol. 8, no. 2, pp. 165-177, 2009. 
[22] X. B. Guo and Z. T. Gong, "Undetermined coefficients method for solving semi-fuzzy matrix equation," in Proceedings of 9th International Conference on Machine Learning and Cybernetics,, vol. 2, pp. 596-600, Qingdao, China, July 2010.

[23] Z. Gong and X. Guo, "Inconsistent fuzzy matrix equations and its fuzzy least squares solutions," Applied Mathematical Modelling, vol. 35, no. 3, pp. 1456-1469, 2011.

[24] X. B. Guo, "Approximate solution of fuzzy Sylvester matrix equations," in Proceedings of the 7th International Conference on Computational Intelligence and Security, pp. 52-57, Sanya, China, December 2011.

[25] X. B. Guo and D. Q. Shang, "Fuzzy symmetric solutions of fuzzy matrix equations," Advances in Fuzzy Systems, vol. 2012, Article ID 318069, 9 pages, 2012.

[26] T. Allahviranloo, M. Ghanbari, A. A. Hosseinzadeh, E. Haghi, and R. Nuraei, "A note on "Fuzzy linear systems"', Fuzzy Sets and Systems, vol. 177, pp. 87-92, 2011.

[27] T. Allahviranloo, F. Hosseinzadeh Lotfi, M. Khorasani Kiasari, and M. Khezerloo, "On the fuzzy solution of $L R$ fuzzy linear systems," Applied Mathematical Modelling. In press.

[28] A. Ben-Israel and T. N. E. Greville, Generalized Inverses:Theory and Applications, Springer, New York, NY, USA, 2nd edition, 2003.

[29] A. Berman and R. J. Plemmons, Nonnegative Matrices in the Mathematical Sciences, Academic Press, New York, NY, USA, 1979.

[30] R. J. Plemmons, "Regular nonnegative matrices," Proceedings of the American Mathematical Society, vol. 39, pp. 26-32, 1973. 


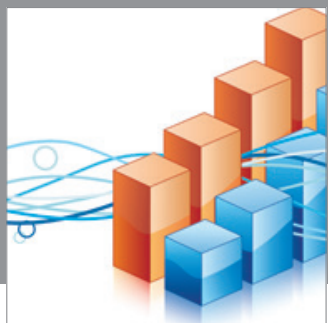

Advances in

Operations Research

mansans

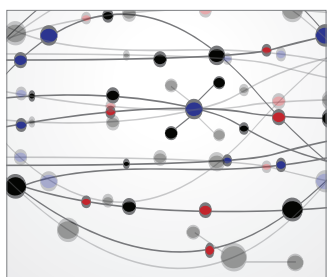

The Scientific World Journal
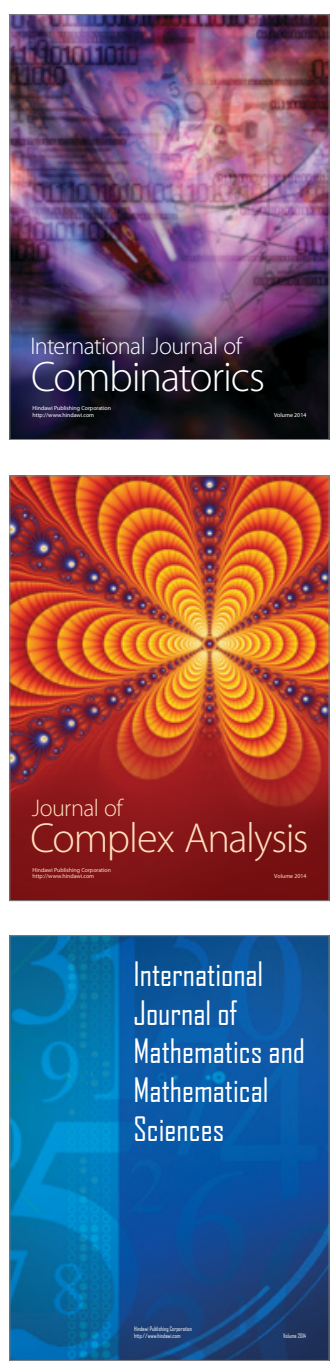
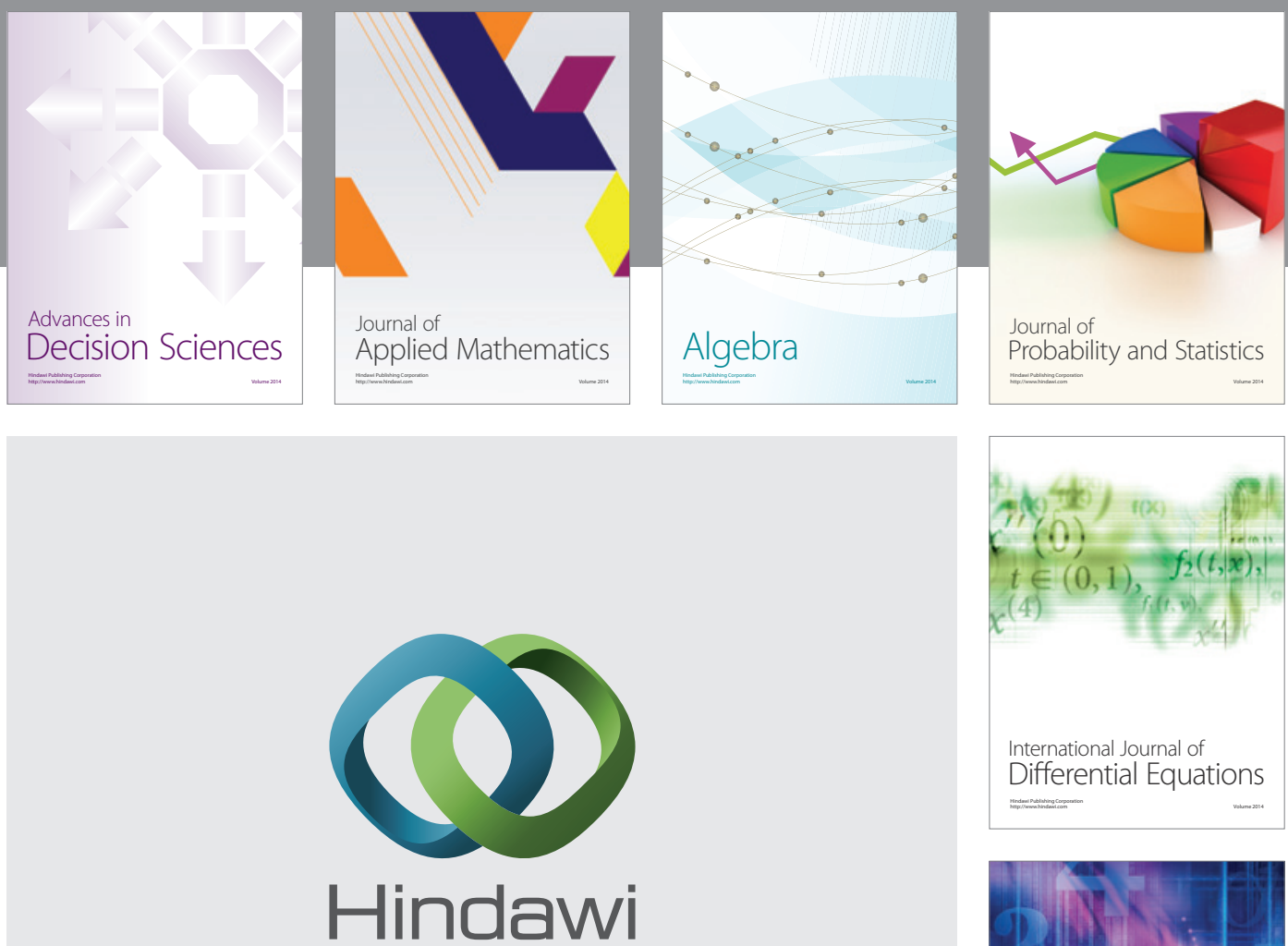

Submit your manuscripts at http://www.hindawi.com
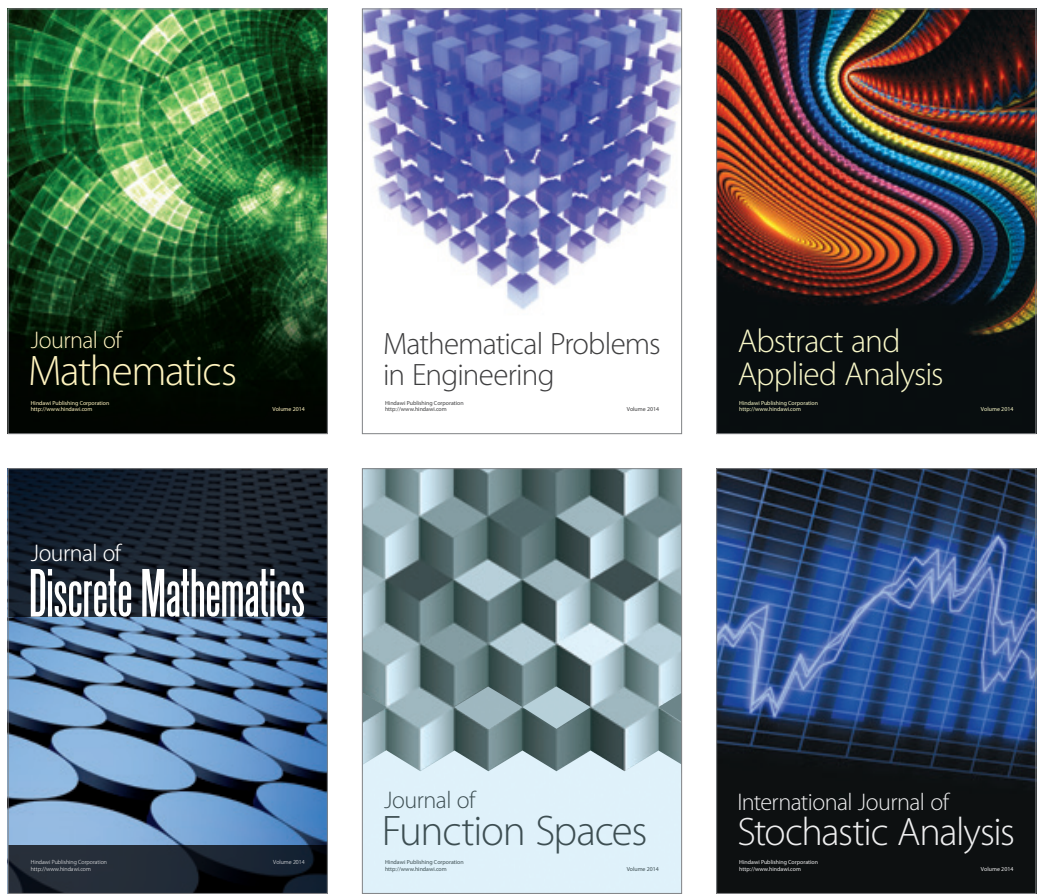

Journal of

Function Spaces

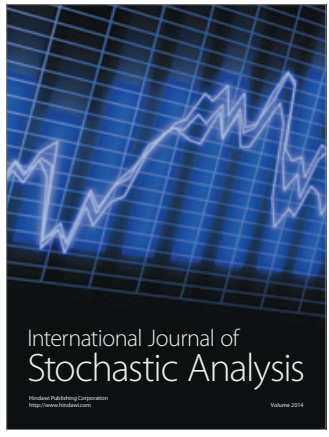

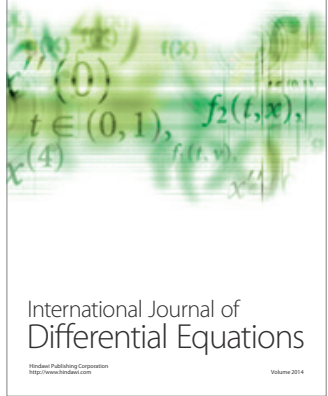
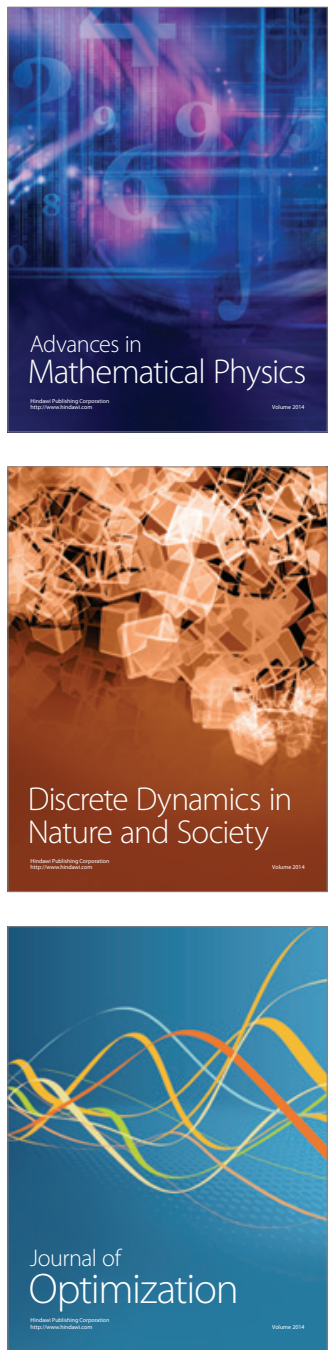